\title{
A Systematic Review of the Risks and Benefits of Venous Thromboembolism Prophylaxis in Traumatic Brain Injury
}

\author{
Joseph Margolick, Charlotte Dandurand, Katrina Duncan, Wenjia Chen, \\ David C. Evans, Mypinder S. Sekhon, Naisan Garraway, \\ Donald E. G. Griesdale, Peter Gooderham, S. Morad Hameed
}

\begin{abstract}
Background: Patients suffering from traumatic brain injury (TBI) are at increased risk of venous thromboembolism (VTE). However, initiation of pharmacological venous thromboprophylaxis (VTEp) may cause further intracranial hemorrhage. We reviewed the literature to determine the postinjury time interval at which VTEp can be administered without risk of TBI evolution and hematoma expansion. Methods: MEDLINE and EMBASE databases were searched. Inclusion criteria were studies investigating timing and safety of VTEp in TBI patients not previously on oral anticoagulation. Two investigators extracted data and graded the papers' levels of evidence. Randomized controlled trials were assessed for bias according to the Cochrane Collaboration Tool and Cohort studies were evaluated for bias using the Newcastle-Ottawa Scale. We performed univariate meta-regression analysis in an attempt to identify a relationship between VTEp timing and hemorrhagic progression and assess study heterogeneity using an $I^{2}$ statistic. Results: Twenty-one studies were included in the systematic review. Eighteen total studies demonstrated that VTEp postinjury in patients with stable head computed tomography scan does not lead to TBI progression. Fourteen studies demonstrated that VTEp administration 24 to 72 hours postinjury is safe in patients with stable injury. Four studies suggested that administering VTEp within 24 hours of injury in patients with stable TBI does not lead to progressive intracranial hemorrhage. Overall, meta-regression analysis demonstrated that there was no relationship between rate of hemorrhagic progression and VTEp timing. Conclusions: Literature suggests that administering VTEp 24 to 48 hours postinjury may be safe for patients with low-hemorrhagic-risk TBIs and stable injury on repeat imaging.
\end{abstract}

RÉSUMÉ: Revue systématique des risques et des bénéfices de la prophylaxie des tromboembolies veineuses chez les patients atteints d'une lésion cérébrale traumatique. Contexte: Les patients qui ont subi une lésion cérébrale traumatique (LCT) présentent un risque accru de tromboembolie veineuse (TEV). Cependant, l'amorce de la tromboprophylaxie veineuse pharmacologique (TEVp) peut aggraver une hémorragie intracrânienne. Nous avons revu la littérature afin de déterminer le moment après le traumatisme où la TEVp peut être administrée sans risque de provoquer une évolution de la TEV et une expansion de l'hématome. Méthodologie: Nous avons effectué une recherche dans MEDLINE et dans EMBASE pour identifier des études sur le moment où la TEVp avait été administrée et la sécurité de la TEVp chez des patients atteints d'un LTC qui ne prenaient pas d'anticoagulants oraux antérieurement. Deux chercheurs ont recueilli les données et évalué le niveau de preuve. Le Cochrane Collaboration Tool a été utilisé pour détecter les biais dans les études contrôlées et randomisées et l'échelle de Newcastle-Ottawa a été utilisée pour évaluer les biais dans les études de cohorte. Nous avons eu recours à une analyse de méta-régression univariée pour tenter d'identifier une relation entre le moment où la TEVp avait été administrée et la progression de l'hémorragie et nous avons évalué l'hétérogénéité des études au moyen de la statistique $I^{2}$. Résultats: Vingt-et-une études ont été incluses dans cette revue systématique. Dans dix-huit études, la TEVp administrée après le traumatisme chez des patients dont le scan à la tomodensitométrie de la tête était stable n'avait pas provoqué de progression de la LTC. Selon 14 études, l'administration de la TEVp 24 à 72 heures après le traumatisme est sûre chez les patients dont la lésion est stable. Quatre études suggéraient que l'administration de la TEVp dans les 24 heures de la blessure, chez les patients dont la LTC est stable, n'entraîne pas de progression d'une hémorragie intracrânienne. Globalement, l'analyse de méta-régression a démontré qu'il n'existait pas de relation entre le taux de progression de l'hémorragie et le moment d'administration de la TEVp. Conclusions: Selon la littérature, la TEVp peut être administrée sans danger 24 à 48 heures après un traumatisme chez les patients dont le risque d'hémorragie est faible et la lésion s'avère stable à l'imagerie.

Key words: Venous thromboembolism, Head Trauma, Chemoprophylaxis

doi:10.1017/cjn.2017.275

Can J Neurol Sci. 2018; 45: 432-444

From the From the Department of Surgery, Divisions of General Surgery (JM, KD, DCE, NG, SMH); Neurosurgery (CD, PG); Department of Medicine, Division of Critical Care (DEGG, MSS), Faculty of Pharmaceutical Sciences (WC ${ }^{4}$, University of British Columbia, Vancouver, British Columbia, Canada.

Received December 20, 2016. Final Revisions Submitted September 6, 2017. Date of Acceptance September 18, 2017.

Correspondence to: S. Morad Hameed, Department of Surgery, University of British Columbia, Trauma Services 855 W 12th Ave Vancouver, BC, V5Z 1M9. Email: morad.hameed@ vch.ca. 
Traumatic brain injury (TBI) is associated with 50,000 deaths and nearly 300,000 hospitalizations annually in the United States, ${ }^{1}$ and is the worldwide leading cause of mortality in patients between the ages of 25 and $44 .{ }^{2}$ There is also a significant social cost associated with brain injury because more than 5 million Americans live with long-term neurological sequelae stemming from $\mathrm{TBI}^{3}$

Patients with TBI are at an increased risk of venous thromboembolism (VTE), defined as either deep vein thrombosis (DVT) or pulmonary embolism (PE). Studies have reported a baseline VTE risk of approximately 5\% in all hospitalized patients ${ }^{4}$; however, for TBI patients, the risk of DVT is approximately $30 \%$ to $60 \%{ }^{2,5-12}$ DVT can pose significant danger to patients because roughly $20 \%$ to $30 \%$ of calf DVTs will extent proximally to the thigh if left untreated, of which approximately $40 \%$ will result in PE. ${ }^{13}$ Overall, PEs occur in $1 \%$ to $24 \%$ of trauma patients. , $7,14,15$ Furthermore, DVTs may cause postthrombotic syndrome and chronic venous stasis, which can significantly affect patients' quality of life. ${ }^{8}$ The exact etiology of the more than fourfold increased rate of DVTs in TBI patients is not entirely clear ${ }^{11}$; however it is thought that TBIs exert a dampening effect on the physiologic dynamics of fibrinolysis, which, when coupled with the immobility caused by prolonged hospitalization creates a highly pro-thrombotic state. ${ }^{16-18}$

Pharmacological venous thromboprophylaxis (VTEp) is effective in reducing rates of VTE ${ }^{2,17}$; however, some literature suggests that VTEp increases the risk of intracranial hemorrhage (ICH) progression ${ }^{19,20}$; therefore, many physicians are hesitant to administer VTEp for fear of causing secondary progression of TBI. ${ }^{17}$ Unfortunately, this delay may cause undue risk from VTE. Clinical decisions must therefore balance the risk of VTE with the potential to cause iatrogenic ICH progression.

There is currently insufficient research to produce formal clinical guidelines on optimal timing of VTEp. ${ }^{9,21}$ In 2002, the Eastern Association for the Surgery of Trauma (EAST) recommended that the safety of VTEp, both low-molecular-weight heparin (LMWH) and unfractionated heparin (UFH) in TBI was not established and VTEp administration should be tailored to each patient. ${ }^{22}$ Similarly, both the American College of Chest Physicians (ACCP) and Brain Trauma Foundation (BTF) recommend VTEp in lieu of mechanical prophylaxis as soon as possible after sustaining a TBI, but could not recommend a specific time frame within which VTEp can be safely administered. ${ }^{23,24}$

Clinical decision tools for VTE prevention improve VTEp adherence, and the ACCP guidelines recommend formal VTE prevention protocols for hospitalized patients. ${ }^{23}$ With this in mind, we set out to review the literature to determine the risk of TBI expansion after VTEp with the ultimate goal of determining how long clinicians should wait after TBI before administering VTEp. A secondary objective of this research was to lay the foundation for a clinical practice guideline (CPG) directing timing of VTEp therapy in TBI patients.

\section{MeTHODS}

The primary objective of this study is to determine the safety of administering VTEp (LMWH or UFH) to TBI patients, and in particular, assessing if VTEp causes intracranial hemorrhagic expansion. The outcome of interest is whether VTEp causes hemorrhagic progression in TBI patients, and if so, within what time frame postinjury. The population of interest includes all hospitalized patients with TBI who were not on anticoagulation preinjury or known to have a preexisting coagulopathy. This systematic review was reported in accordance with the Preferred Reporting of Items for Systematic Reviews and Meta-Analyses protocols. ${ }^{25}$ The literature was searched using databases MEDLINE and EMBASE. We limited our findings to English language studies published between January 1, 1999, and December 1, 2015. A combination or partial combinations of the following search terms were used: traumatic brain injury, venous thromboembolism, anticoagulant agent, anticoagulants, fibrinolytic agent, prophylaxis, thromboprophylaxis, chemoprophylaxis, brain injuries, and venous thrombosis. (Please see the supplementary appendix for detailed search strategy.) Animal studies, articles about patients taking anticoagulants before TBI and articles on nontraumatic intracranial hemorrhage (hemorrhagic stroke) were excluded. Articles with short titles such a "Discussion" or "Author Reply" and those without an abstract were excluded. Titles of articles were first reviewed for relevance. Abstracts of selected articles were then reviewed for inclusion in the study. Two independent investigators (JM and CD) then evaluated full texts of selected articles. Reference sections of the articles were reviewed to identify additional relevant studies.

Two investigators (JM and CD) independently extracted data from the included studies and assessed the methodological quality based on the Oxford Centre for Evidence Based Medicine Levels of Evidence. ${ }^{26}$ Each study was given an evidence class score ranging from 1a (high-quality randomized controlled trials [RCTs]) to 4 (case series and poor-quality case-cohort studies). A score of $1 \mathrm{~b}$ was given to RCTs with narrow confidence intervals, 2a to systematic reviews with homogeneity, $2 \mathrm{~b}$ to low-quality RCTs and retrospective cohort studies, 3 a to systematic reviews with heterogeneity or case control studies, and $3 \mathrm{~b}$ to individual case control studies. Data were extracted from the included studies using a standardized form. We collected authors, date of publication, journal of publication, a descriptive evaluation of each study, research design, timing of VTEp, study limitations, and main findings (Table 1). RCTs were assessed for bias based on the Cochrane Collaboration Tool ${ }^{27}$ and cohort studies were evaluated for bias using the Newcastle-Ottawa Scale. ${ }^{28}$

Statistical analyses were performed using Stata 12.1 software package (College Station, TX). Pooled rate estimates from the studies were plotted against VTEp timing (i.e. no VTEp vs VTEp given within 24 hours of injury, 48 hours of injury, 72 hours of injury, and $>72$ hours after injury). We performed univariate metaregression analysis in an attempt to identify a relationship between VTEp timing and hemorrhagic progression and assess study heterogeneity (variance between studies) using an $I^{2}$ statistic.

After the formal literature review, a consultation process with two trauma surgeons, two intensivists, and a neurosurgeon was carried out to review the literature findings. The consultation process occurred over several meetings and involved reviewing all available literature. Expert opinion, based on years of clinical work in trauma, critical care, and neurosurgery, was used to supplement the data from the review and to attain consensus opinion on a CPG.

\section{RESULTS}

Eighty-one articles from the MEDLINE database and 332 from the EMBASE database were selected for abstract review 


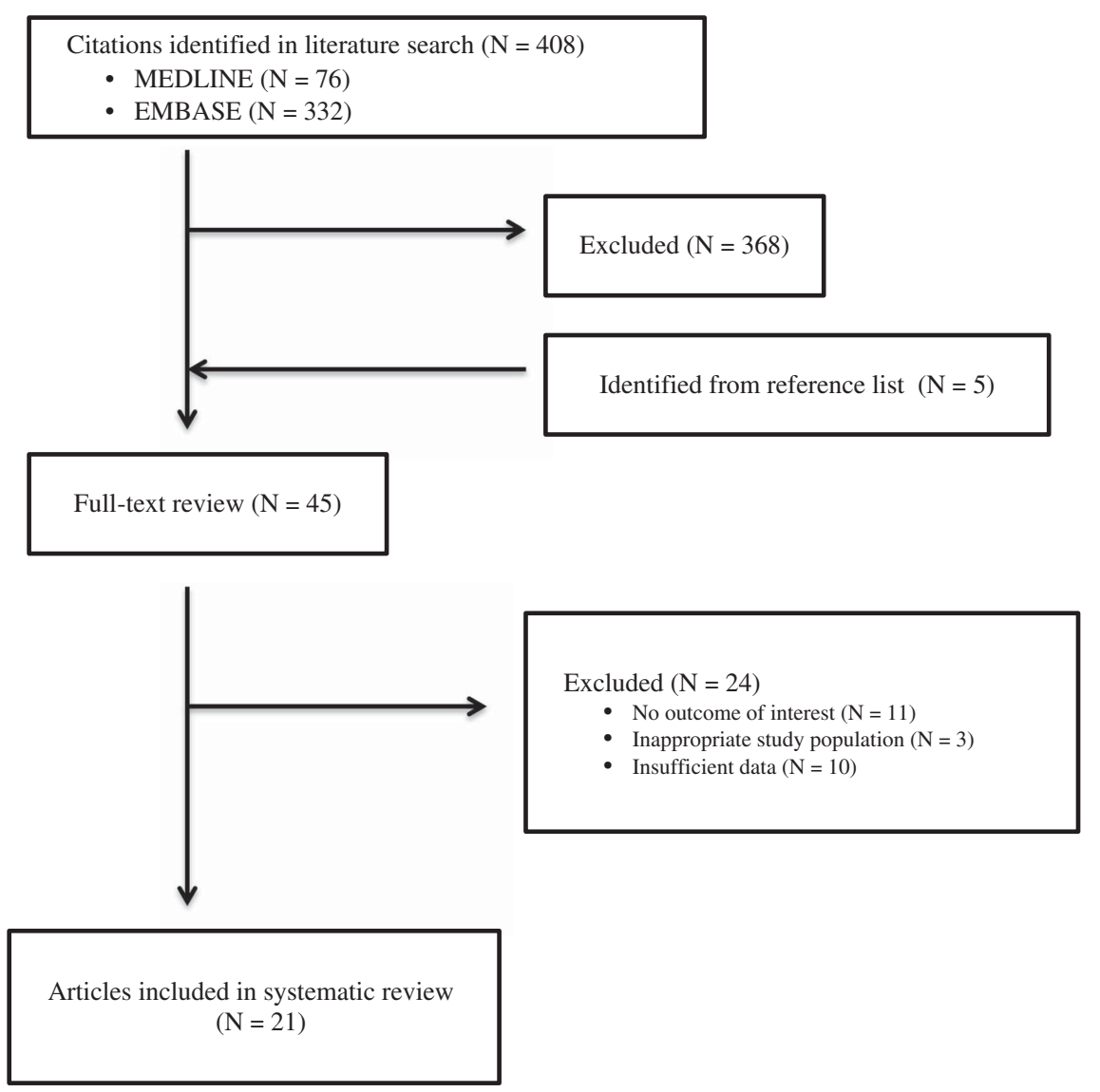

Figure 1: Methods Flow Diagram

(Figure 1). Forty-five articles were reviewed in entirety, of which 21 were included in the systematic review. Reasons for exclusion after full review were: no data regarding VTEp ${ }^{29,30}$ data from rehabilitation centers, ${ }^{31,32}$ no data on TBI progression, ${ }^{11,13,20,21,33,34}$ investigating patients on oral anticoagulation before arriving in the hospital, ${ }^{35}$ published critique of previous article ${ }^{36}$ literature reviews of studies already captured by our review, ${ }^{2,3,8,9,12,18,37-39}$ no data on timing of VTEp, ${ }^{40}$ and a study investigating inferior vena cava (IVC) filters. ${ }^{14}$

Eighteen total studies demonstrated that VTEp postinjury in patients with stable head computed tomography (CT) scan does not lead to radiographic or clinical TBI progression. Fourteen studies demonstrated that VTEp administration specifically 24 to 72 hours postinjury is safe in patients with stable injury. Four studies suggested that administering VTEp specifically within 24 hours of injury in patients with stable TBI does not lead to progressive ICH. However, one study, a retrospective review with multivariate analysis of 1215 patients, suggested LMWH is a risk factor for TBI progression, defined as "progressive bleed on follow up CT scan," 41 and another retrospective review found UFH was associated with higher rates of TBI progression, defined as worsening bleeding on repeat CT head, than LMWH. ${ }^{42}$ A study by Lin et al divided TBIs into those with ICH and without ICH. They determined that the rate of ICH progression when VTEp was administered within 48 hours was $10.6 \%$ in those with preexisting ICH compared with $0.7 \%$ in those without $\mathrm{ICH}{ }^{43}$

Meta-regression analysis of the studies demonstrated that there was no relationship between rate of hemorrhagic progression and
VTEp timing. Overall, the timing of DVT prophylaxis only explains $1.56 \%$ of the variations between rebleeding rates reported in different studies, which is not statistically significant $(p=0.31)$. Because of the high level of heterogeneity (variation between studies, $I^{2}>95 \%$ ) detected by the meta-analysis, we did not report weighted-pooled summary estimates for rebleeding rate in this review. Four of 12 studies directly comparing VTE rates between early and late VTEp administration reported a significant decrease in VTE rates with early VTEp administration. ${ }^{17,41,46,47}$ Similarly, another seven studies found VTEp rates of only $0.0 \%$ and $2.0 \%$ in patients given VTEp within 24 to 72 hours of injury. Three other retrospective reviews found somewhat higher rates of VTE that ranged from $7.3 \%$ to $14 \% .^{21,54,57}$ Summary of literature can be found in Table 1; see Table 2 for risk of bias assessment of RCTs based on the Cochrane Collaboration tool. ${ }^{26}$ Only seven of the 18 nonrandomized cohort studies reviewed were deemed "low risk" for bias according to Newcastle-Ottawa Scale (Table 3). ${ }^{27} \mathrm{~A}$ preliminary CPG was formulated based on data from this review and consensus opinion among clinical experts (Figure 2).

\section{DISCUSSION}

TBI is a common cause of death and disability in young patients. $^{2}$ Preventing secondary brain injury is the primary objective of TBI management, yet complications of VTE can be devastating. Clinicians should be weary of iatrogenic hemorrhagic progression secondary to VTEp; however, delaying chemoprophylaxis may cause unwarranted increases in morbidity and 


\begin{tabular}{|c|c|c|c|c|c|c|}
\hline Manuscript & EC & Research design & Timing of VTEp & TBI progression & Limitations & Findings and conclusion \\
\hline $\begin{array}{l}\text { Kim et al, } 2014^{1} \\
N=75\end{array}$ & $2 b$ & $\begin{array}{c}\text { Retrospective } \\
\text { cohort study }\end{array}$ & $\begin{array}{l}\text { Early: }<72 \text { hours postadmission } \\
\text { Intermediate: } 72-120 \text { hours } \\
\text { Late: }>120 \text { hours post admission } \\
\text { LMWH or UFH }\end{array}$ & $\begin{array}{l}\text { Head CT scans from all three groups } \\
\text { worsened during first } 24 \text { hours, } \\
\text { but stabilized thereafter. Early } \\
\text { VTEp increased rate of } \\
\text { radiographic stabilization }\end{array}$ & $\begin{array}{l}\text { Retrospective } \\
\text { Small sample size }\end{array}$ & $\begin{array}{l}\text { Early VTEp group improved clinically faster than } \\
\text { the late group ( } \mathrm{p}<0.05 \text { ) } \\
\text { VTEp increases rate of TBI stabilization when } \\
\text { given within } 72 \text { hours of injury } \\
\text { No difference in TBI severity (radiographic or } \\
\text { clinical) between groups }\end{array}$ \\
\hline $\begin{array}{l}\text { Faroooqui et al, } \\
2013^{46} \\
\mathrm{~N}=236\end{array}$ & $2 \mathrm{~b}$ & $\begin{array}{l}\text { Retrospective } \\
\text { cohort study }\end{array}$ & $\begin{array}{l}\text { Preprotocol group: no protocol, } \\
\text { VTEp based on individual clinical } \\
\text { decision } \\
\text { Protocol group: VTEp within } \\
24 \text { hours of admission if stable } \\
\text { repeat CT scan of the head } \\
\text { LMWH or UFH }\end{array}$ & $\begin{array}{l}\text { 3/107 (2.8\%) had progression of } \\
\text { ICH during preprotocol study } \\
\text { period } \\
\text { 1/129 }(0.7 \%) \text { had progression of } \\
\text { ICH with early VTEp protocol }\end{array}$ & $\begin{array}{l}\text { Retrospective } \\
\text { Protocol noncompliance }\end{array}$ & $\begin{array}{l}\text { No significant difference in progression of ICH } \\
\text { with protocol: } 0.7 \% \text { vs } 2.8 \% \text { preprotocol). } \\
\text { DVT rates lower in protocol group lower }(0.0 \%) \\
\text { compared with preprotocol group }(5.6 \%, \mathrm{p}<0 \\
.05) \\
\text { VTEp within } 24 \text { hours of admission and stable } \\
\text { TBI is safe and effective }\end{array}$ \\
\hline $\begin{array}{l}\text { Jamjoom and } \\
\text { Jamjoom, } 2013^{17} \\
\mathrm{~N}=1624 \\
\text { (5 studies) }\end{array}$ & $2 \mathrm{a}$ & Metaanalysis & $\begin{array}{l}\text { Early: }<72 \text { hours postadmission } \\
\text { Late: }>72 \text { hours postadmission } \\
\text { LMWH or UFH }\end{array}$ & $\begin{array}{l}\text { Pooled data of three studies } \\
\text { demonstrated that early VTEp had } \\
\text { RR and } 95 \% \text { CI of } 0.64(0.35- \\
\text { 1.14) for developing TBI } \\
\text { progression }\end{array}$ & $\begin{array}{l}\text { Heterogeneous studies } \\
\text { Selection bias: patients with severe } \\
\text { ICH or progression included in } \\
\text { late group }\end{array}$ & $\begin{array}{l}\text { Patients in the early group had almost half the } \\
\text { number of VTEs, with RR of } 0.52 \\
\text { No difference in ICH progression between early } \\
\text { and late groups }\end{array}$ \\
\hline $\begin{array}{l}\text { Nickele et al, 2012 } \\
\quad \mathrm{N}=135\end{array}$ & $2 \mathrm{~b}$ & Cohort study & $\begin{array}{l}\text { Control: Mean time to } \\
\text { VTEp }=4.9 \text { days postadmission. } \\
\text { Individual clinical decisions } \\
\text { Protocol: VTEp } 24 \text { hours after stable } \\
\text { repeat head CT scans (mean time } \\
\text { to VTEp, } 3.4 \text { days) } \\
\text { LMWH or UFH }\end{array}$ & No VTEp related progression of ICH & $\begin{array}{l}\text { Not randomized } \\
\text { Protocol non- compliance } \\
\text { Small sample size }\end{array}$ & $\begin{array}{l}\text { No difference between control and protocol group } \\
\text { in incidence of DVT ( } 4.2 \% \text { vs } 6.9 \% \text { ) or PEs } \\
\text { ( } 4.2 \% \text { vs } 5.75 \%) \\
\text { No patients required their VTEp to be stopped due } \\
\text { to TBI expansion. No significant difference in } \\
\text { ICH progression between control and protocol } \\
\text { groups. }\end{array}$ \\
\hline $\begin{array}{l}\text { Phelan et al. } 2012^{51} \\
\quad \mathrm{~N}=62\end{array}$ & $2 b$ & $\begin{array}{l}\mathrm{RCT} \\
\text { noninferiority } \\
\text { trial }\end{array}$ & $\begin{array}{l}\text { Control: placebo SC BID } 24 \text { hours } \\
\text { after stable repeat CT scan of the } \\
\text { head } \\
\text { Intervention: Enoxaparin } 30 \mathrm{mg} \mathrm{SC} \\
\text { BID } 24 \text { hours after stable repeat } \\
\text { CT scans of the head }\end{array}$ & $\begin{array}{l}5.9 \% \text { of patients had radiographic } \\
\text { progression on scans performed } \\
24 \text { hours after commencement of } \\
\text { VTEp vs } 3.6 \% \text { in the placebo } \\
\text { group (not significant) }\end{array}$ & $\begin{array}{l}\text { Small sample size } \\
\text { No routine VTE screening with } \\
\text { Doppler ultrasound }\end{array}$ & $\begin{array}{l}\text { No significant difference in radiographic TBI } \\
\text { progression compared with control } \\
\text { Any radiographic progression seen was not } \\
\text { associated with clinical progression of TBI } \\
\text { One patient }(3.5 \%) \text { had DVT in placebo group }\end{array}$ \\
\hline $\begin{array}{l}\text { Saadeh et al, } 2012^{10} \\
\quad \mathrm{~N}=122\end{array}$ & $2 b$ & $\begin{array}{l}\text { Retrospective case } \\
\text { series }\end{array}$ & $\begin{array}{l}\text { Early: VTEp started } 24-48 \text { hours } \\
\text { after stable CT scan of the head } \\
\text { Late: VTEp started }>48 \text { hours after } \\
\text { stable CT scan of the head } \\
\text { LMWH or UFH }\end{array}$ & $\begin{array}{l}\text { No patients who received VTEp } \\
(\mathrm{n}=93) \text { had progression of ICH }\end{array}$ & $\begin{array}{l}\text { Retrospective } \\
\text { Small sample size }\end{array}$ & $\begin{array}{l}\text { No patients developed VTE } \\
\text { VTEp within } 24-48 \text { hours of a radiographically } \\
\text { stable TBI is safe }\end{array}$ \\
\hline $\begin{array}{l}\text { Minshall et al, } \\
2011^{42} \\
\mathrm{~N}=386\end{array}$ & $2 b$ & $\begin{array}{l}\text { Retrospective } \\
\text { cohort study }\end{array}$ & $\begin{array}{l}\text { VTEp initiated once stable CT of the } \\
\text { head is complete } \\
\text { UFH } 5000 \text { U TID: Mean time to } \\
\text { initiation was } 47 \text { hours } \\
\text { postadmission } \\
\text { LMWH (enoxaparin } 30 \mathrm{mg} \text { BID): } \\
\text { Mean time to initiation was } \\
54 \text { hours postadmission }\end{array}$ & $\begin{array}{l}\text { LMWH group: rate of ICH } \\
\text { progression was } 5 \% \\
\text { UFH group: rate of ICH progression } \\
\text { was } 12 \%\end{array}$ & $\begin{array}{l}\text { Retrospective } \\
\text { Selection bias: head AIS and ISS } \\
\text { significantly higher in UFH group } \\
8 \% \text { of patients in each group had } \\
\text { progression of ICH before } \\
\text { initiation of VTEp }\end{array}$ & $\begin{array}{l}\text { UFH group had significantly more PEs }(3.7 \%) \\
\text { than LMWH group }(0.0 \%), p<0.05 \text {. Rates of } \\
\text { isolated DVT were } 1 \% \text { in each group } \\
\text { Higher rate of ICH progression in the UFH group } \\
\text { than the LMWH group }(\mathrm{p}<0.05)\end{array}$ \\
\hline $\begin{array}{l}\text { Scudday et al, } \\
2011^{47} \\
\mathrm{~N}=812\end{array}$ & $2 b$ & $\begin{array}{l}\text { Retrospective } \\
\text { cohort study }\end{array}$ & $\begin{array}{l}\text { Prophylaxis group: VTEp within 24- } \\
72 \text { of stable CT scan of the head } \\
\text { No prophylaxis group: no VTEp } \\
\text { LMWH or UFH }\end{array}$ & $\begin{array}{l}6 \% \text { in the no prophylaxis group had } \\
\text { TBI progression versus } 3 \% \text { in the } \\
\text { prophylaxis group, } \\
\mathrm{p}=0.05\end{array}$ & $\begin{array}{l}\text { Retrospective } \\
\text { Variability of VTEp timing } \\
\text { Routine screening of VTE in the no } \\
\text { prophylaxis group only } \\
\text { Selection bias: prophylaxis group } \\
\text { significantly more injured }\end{array}$ & $\begin{array}{l}\text { The prophylaxis group was more injured (ISS of } \\
23.8 \text { vs } 16.6, \mathrm{p}<0.01) \text { and had more patients } \\
\text { with GCS }<9(185 \mathrm{vs} 115, \mathrm{p}<0.01) . \\
\text { Patients in the prophylaxis group had } \\
\text { significantly fewer VTEs }(1.0 \% \text { vs } 3.0 \%, \mathrm{p}< \\
0.05 \text { ) } \\
\text { VTEp safe and effective }\end{array}$ \\
\hline
\end{tabular}


Table 1. Continued

\begin{tabular}{|c|c|c|c|c|c|c|}
\hline Manuscript & EC & Research design & Timing of VTEp & TBI progression & Limitations & Findings and conclusion \\
\hline $\begin{array}{l}\text { Kwiatt et al, } 2012^{41} \\
\quad \mathrm{~N}=1215\end{array}$ & $2 \mathrm{~b}$ & $\begin{array}{l}\text { Retrospective } \\
\text { cohort study }\end{array}$ & $\begin{array}{l}\text { Early: VTEp }<48 \text { hours } \\
\text { postadmission } \\
\text { Intermediate: } 48 \text { hours- } 7 \text { days } \\
\text { postadmission } \\
\text { Late: }>7 \text { days postadmission } \\
\text { No prophylaxis }\end{array}$ & $\begin{array}{l}\text { TBI progression } \\
\text { Early: } 22.2 \% \\
\text { Intermediate: } 12.6 \% \\
\text { Late: } 14.9 \% \\
\text { No VTEp: } 24 \%\end{array}$ & $\begin{array}{l}\text { Retrospective } \\
\text { Selection bias: LMWH group had } \\
\text { more severe injury on admission } \\
\text { than no prophylaxis group }\end{array}$ & $\begin{array}{l}\text { Timing of LMWH did not affect TBI progression } \\
10 \% \text { of patients had TBI progression after VTEp } \\
\text { LMWH strongest risk factor for TBI progression } \\
\text { (OR, } 2.41 ; 95 \% \mathrm{CI}, 1.65-3.53)\end{array}$ \\
\hline $\begin{array}{l}\text { Salottolo et al, } \\
2011^{52} \\
\mathrm{~N}=480\end{array}$ & $2 \mathrm{~b}$ & $\begin{array}{l}\text { Retrospective } \\
\text { cohort study }\end{array}$ & $\begin{array}{l}\text { Early: initiation of VTEp }<72 \text { hours } \\
\text { postadmission } \\
\text { Late: Initiation of VTEp }>72 \text { hours } \\
\text { postadmission } \\
\text { LMWH or UFH }\end{array}$ & $\begin{array}{l}\text { Early VTEp group: } 6.5 \% \text { rate of TBI } \\
\text { progression } \\
\text { Late VTEp group: } 14.3 \% \text { rate of TBI } \\
\text { progression } \\
\text { No VTEp group: } 8.5 \% \text { rate of TBI } \\
\text { progression } \\
\end{array}$ & $\begin{array}{l}\text { Retrospective } \\
\text { Unclear definition of TBI } \\
\text { progression }\end{array}$ & $\begin{array}{l}\text { TBI progression did not differ between patients } \\
\text { receiving early, late or no VTEp, } \mathrm{p}=0.92 \\
15 \text { patients }(3.1 \%) \text { developed VTE. Neither VTEp } \\
\text { administration nor timing of VTEp were } \\
\text { independent predictors of VTE }\end{array}$ \\
\hline $\begin{array}{l}\text { Callcut et al, } 2011^{53} \\
\quad \mathrm{~N}=77\end{array}$ & $2 b$ & $\begin{array}{l}\text { Retrospective case } \\
\text { series }\end{array}$ & $\begin{array}{l}\text { Therapeutic IV UFH or ASA started } \\
\text { on median day } 3 \text { after admit } \\
\text { (roughly } 24-48 \text { hours after stable } \\
\text { repeat CT scan of the head) } \\
\text { Treated group: ASA or therapeutic } \\
\text { UFH } \\
\text { Untreated group: SC prophylactic } \\
\text { UFH or no treatment }\end{array}$ & $\begin{array}{l}\text { No significant difference in } \\
\text { radiographic TBI progression } \\
\text { between treated group }(5.1 \%) \text { and } \\
\text { untreated group }(6.3 \%)\end{array}$ & $\begin{array}{l}\text { Retrospective } \\
\text { VTE rates not measured } \\
\text { Small sample size } \\
\text { VTEp with SC UFH considered in } \\
\text { the untreated group }\end{array}$ & $\begin{array}{l}\text { Rates of TBI progression not significantly } \\
\text { different in patients treated with IV UFH, ASA, } \\
\text { VTEp, or no VTEp }\end{array}$ \\
\hline $\begin{array}{l}\text { Koehler et al, } \\
2011^{49} \\
\mathrm{~N}=669\end{array}$ & $2 \mathrm{~b}$ & $\begin{array}{l}\text { Retrospective } \\
\text { cohort study }\end{array}$ & $\begin{array}{l}\text { Early: VTEp }<72 \text { hours } \\
\text { postadmission (mean, } 2.7 \text { days) } \\
\text { Late: VTEp }>72 \text { hours (mean, } \\
5.3 \text { days) }\end{array}$ & $\begin{array}{l}\text { 1.5\% had TBI progression after } \\
\text { VTEp in early group } \\
1.5 \% \text { had TBI progression after } \\
\text { VTEp in late group }\end{array}$ & $\begin{array}{l}\text { Retrospective } \\
\text { Selection bias: rates of TBI } \\
\text { progression before initiation of } \\
\text { VTEp was significantly higher in } \\
\text { late group }\end{array}$ & $\begin{array}{l}1.5 \% \text { of patients developed VTEs in early group } \\
\text { versus } 3.5 \% \text { in late group, } p=0.117 \\
\text { No significant difference in ICH progression } \\
\text { between early and late VTEp groups across all } \\
\text { injury types }\end{array}$ \\
\hline $\begin{array}{l}\text { Norwood et al, } \\
2008^{6} \\
\mathrm{~N}=525\end{array}$ & 3 & $\begin{array}{l}\text { Prospective } \\
\text { observational }\end{array}$ & $\begin{array}{l}\text { VTEp group: all patients received } \\
\text { VTEp within } 48 \text { hours of } \\
\text { admission (mean time, } \\
36.2 \text { hours) } \\
\text { Enoxaparin } 30 \mathrm{mg} \mathrm{BID}\end{array}$ & $\begin{array}{l}\text { Progressive hemorrhage in } 3.4 \% \text { if } \\
\text { patients after administering VTEp } \\
8.3 \% \text { of patients had ICH } \\
\text { progression at baseline } \\
\text { Had there been no protocol } \\
\text { violations, rate of TBI progression } \\
\text { after VTEp would have been } \\
1.8 \% \\
56 \% \text { of TBI progressions resulting } \\
\text { from protocol violations }\end{array}$ & $\begin{array}{l}\text { Nonrandomized, observational } \\
\text { Protocol violations: } 5 \text { of } 6 \text { patients } \\
\text { with clinically significant TBI } \\
\text { progression were due to protocol } \\
\text { violations }\end{array}$ & $\begin{array}{l}\text { VTE rate of } 1.14 \% \text { (no PEs) } \\
12 \text { of } 18 \text { patients who had TBI progression were } \\
\text { considered clinically insignificant } \\
\text { If protocol followed closely, VTEp can be } \\
\text { administered within } 48 \text { hours of admission with } \\
\text { low risk of clinically significant TBI } \\
\text { progression }\end{array}$ \\
\hline $\begin{array}{l}\text { Dudley et al, } 2010^{54} \\
\mathrm{~N}=287\end{array}$ & $2 b$ & $\begin{array}{l}\text { Retrospective } \\
\text { cohort study }\end{array}$ & $\begin{array}{l}\text { VTEp } 48-72 \text { hours after injury if } \\
\text { stable repeat head CT scan } \\
\text { Enoxaparin } 30 \mathrm{mg} \mathrm{BID*} \text { Or } \\
\text { dalteparin } 5000 \mathrm{U} \text { daily }\end{array}$ & $\begin{array}{l}1 \text { patient }(0.4 \%) \text { had TBI } \\
\text { progression } 2 \text { weeks } \\
\text { postadmission }\end{array}$ & $\begin{array}{l}\text { Retrospective } \\
\text { Follow-up CT scans were not done } \\
\text { systematically; only if clinically } \\
\text { warranted }\end{array}$ & $\begin{array}{l}\text { VTE rate of } 7.3 \% \text { ( } 3.1 \% \text { proximal DVTs) } \\
\text { No difference in VTE rate between enoxaparin } \\
\text { and dalteparin (7.0 vs } 7.5 \% \mathrm{p}=0.87 \text { ) } \\
\text { Very low risk of TBI progression after early } \\
\text { administration of VTEp }\end{array}$ \\
\hline $\begin{array}{l}\text { Lin et al, } 2013^{43} \\
\mathrm{~N}=3812\end{array}$ & $2 b$ & $\begin{array}{l}\text { Retrospective } \\
\text { cohort study }\end{array}$ & $\begin{array}{l}\text { Within } 48 \text { hours of injury if stable } \\
\text { CT head } \\
\text { UFH }\end{array}$ & $\begin{array}{l}\text { 10.6\% of patients with ICH had } \\
\text { radiographic progression if given } \\
\text { UFH within } 48 \text { hours } \\
0.7 \% \text { of patients with TBI without } \\
\text { ICH had radiographic progression } \\
\text { (developed ICH) if given UFH } \\
\text { within } 48 \text { hours }\end{array}$ & $\begin{array}{l}\text { Retrospective } \\
\text { Poor protocol compliance } \\
\text { Did not define ICH or TBI } \\
\text { progression } \\
\text { Dosing of UFH not in accordance } \\
\text { with North American norms } \\
(5000 \text { U TID }) \dagger\end{array}$ & $\begin{array}{l}\text { UFH protocol did not decrease incidence of VTE } \\
\text { ( } 0.97 \% \text { vs } 1.24 \%, \mathrm{p}=0.492) \\
\text { TBI, defined by AIS }>3 \text { without ICH, has a very } \\
\text { low rate of progression secondary to VTEp } \\
\text { compared with patients with ICH }\end{array}$ \\
\hline
\end{tabular}




\begin{tabular}{|c|c|c|c|c|c|c|c|}
\hline $\begin{array}{c}5 \\
\vdots \\
\vdots \\
0 \\
\vdots \\
0 \\
\vdots \\
0 \\
1 \\
1\end{array}$ & $\begin{array}{l}\text { Norwood et al, } \\
2002^{55} \\
\mathrm{~N}=177\end{array}$ & 3 & $\begin{array}{l}\text { Prospective } \\
\text { observational }\end{array}$ & $\begin{array}{l}\text { VTEp within } 24 \text { hours of admission } \\
\text { Enoxaparin } 30 \mathrm{mg} \text { BID* }\end{array}$ & $\begin{array}{l}6 \text { patients ( } 4 \% \text { ) of patients had ICH } \\
\text { progression after administration } \\
\text { of enoxaparin. Two of the } 6 \\
\text { patients were postoperative } \\
\text { ICH progression found in } 28 \text { patients } \\
(\text { prof) before initiation of VTEp. } \\
\text { Enoxaparin was still given in } \\
\text { these patients and there were no } \\
\text { further ICH progression }\end{array}$ & $\begin{array}{l}\text { No control or comparison group } \\
\text { Protocol violation: } 2 \text { of the } 6 \text { patients } \\
\text { with TBI progression were } \\
\text { protocol violations }\end{array}$ & $\begin{array}{l}\text { DVT rate, 2\% } \\
\text { LMWH does not increase the rate of ICH } \\
\text { progression when given to patients with } \\
\text { radiographically progressing TBI } \\
\text { A significiant decrease in rate of progression of } \\
\text { ICH after } 24 \text { hours and administration of } \\
\text { VTEp, }=0.002\end{array}$ \\
\hline 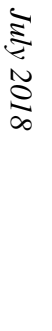 & $\begin{array}{l}\text { Levy et al, } 2010^{19} \\
\mathrm{~N}=340\end{array}$ & $2 \mathrm{~b}$ & $\begin{array}{l}\text { Retrospective } \\
\text { cohort study }\end{array}$ & $\begin{array}{l}\text { Early: VTEp }<72 \text { hours } \\
\text { postadmission } \\
\text { Late: VTEp }>72 \text { hours } \\
\text { postadmission } \\
\text { No traatment: No VTEp } \\
\text { administered } \\
\text { Enoxaparin } 30 \mathrm{mg} \text { BID }\end{array}$ & $\begin{array}{l}\text { 7.3\% of patients with initially stable } \\
\text { head CT scans had progression. } \\
\text { Neither timing nor VTEp } \\
\text { exposure associated with } \\
\text { progression } \\
\text { 92 patients had ICH progression } \\
\text { before initiation of VTEp. The } \\
\text { greatest predictor of further } \\
\text { progression after unstable CT } \\
\text { scan of the head was VTEp } \\
\text { administration (OR, 13.1; } 95 \% \\
\text { CI, } 1.6-103.4 \text { ) }\end{array}$ & $\begin{array}{l}\text { Retrospective } \\
\text { Selection bias: } 60 \% \text { with VTEp had } \\
\text { severe TBI versus } 12 \% \text { in no } \\
\text { treatment group } \\
73 \text { patients had their VTEp stopped } \\
\text { then restarted }\end{array}$ & $\begin{array}{l}\text { Administration of VTEp does not increase risk of } \\
\text { ICH progression in those with a stable CT scan } \\
\text { of the head } \\
\text { Conversely in patients with progressive ICH, } \\
\text { VTEp is associated with 13-fold increased in } \\
\text { odds of developing further ICH progression }\end{array}$ \\
\hline & $\begin{array}{l}\text { Kurtoglu et al, } \\
2004^{56} \\
\mathrm{~N}=120\end{array}$ & $2 \mathrm{~b}$ & RCT & $\begin{array}{l}\text { Determined by neurosurgery and } \\
\text { trauma staff after CT scan of the } \\
\text { head } 24 \text { hours postinjury } \\
\text { Enoxaparin } 40 \mathrm{mg} \text { daily }\end{array}$ & $\begin{array}{l}\text { LMWH group: } 2.0 \% \text { had } \\
\text { progression of ICH } \\
\text { IPC group: } 2.0 \% \text { had progression of } \\
\text { ICH } \ddagger\end{array}$ & $\begin{array}{l}\text { Not truly randomized } \\
\text { Not clear exactly when VTEp started } \\
\text { Enoxaparin dosing not standard for } \\
\text { North America }\end{array}$ & $\begin{array}{l}\text { No difference in ICH progression between } \\
\text { LMWH and IIC group } \\
\text { 6.6\% developed DVT and 3.3\% developed PEs } \\
\text { in IPC group versus 5.0\% and 6.6\% in LMWH } \\
\text { group }\end{array}$ \\
\hline & $\begin{array}{l}\text { Depew et al, } 2008^{21} \\
\mathrm{~N}=124\end{array}$ & $2 \mathrm{~b}$ & $\begin{array}{l}\text { Retrospective } \\
\text { cohort study }\end{array}$ & $\begin{array}{l}\text { Early: VTEp }<72 \text { hours } \\
\text { postadmission } \\
\text { Late: VTEp >72 hours } \\
\text { postadmission } \\
\text { No treatment: No VTEp } \\
\text { LMWH or UFH } \\
\end{array}$ & $\begin{array}{l}\text { Early group: } 3.0 \% \text { TBI progression } \\
\text { Late group: } 4.0 \% \text { TBI progression } \\
\text { No treatment: } 0 \% \text { progression }\end{array}$ & $\begin{array}{l}\text { Retrospective } \\
\text { Selection bias: no between-group } \\
\text { comparison }\end{array}$ & $\begin{array}{l}\text { Early group had VTE rate of } 14 \% \text { versus } 11 \% \text { in } \\
\text { the late group and } 0.0 \% \text { in the no treatment } \\
\text { group } \\
\text { LMWH is safe with stable TBI }\end{array}$ \\
\hline & $\begin{array}{l}\text { Kim et al, } 2002^{57} \\
N=64\end{array}$ & $2 \mathrm{~b}$ & $\begin{array}{l}\text { Retrospective } \\
\text { cohort study }\end{array}$ & $\begin{array}{l}\text { Early: VTEp }<72 \text { hours } \\
\text { postadmission } \\
\text { Late: VTEp >72 hours } \\
\text { postadmission } \\
\text { UFH } 5000 \text { U BID }\end{array}$ & $\begin{array}{l}\text { No patients in early group had TBI } \\
\text { progression }\end{array}$ & $\begin{array}{l}\text { Retrospective } \\
\text { Small sample size } \\
\text { CT scans after initiation of VTEp } \\
\text { were obtained in only } 88 \% \text { of } \\
\text { study population }\end{array}$ & $\begin{array}{l}\text { No significant difference in VTE between early } \\
\text { and late groups }(8.5 \% \text { vs } 6 \%) \\
\text { Early VTEp pis safe and does not exacerbate } \\
\text { intracranial injury }\end{array}$ \\
\hline
\end{tabular}

AIS = Abbreviated Injury Score; ASA = aspirin; $\mathrm{BID}=2$ times daily; $\mathrm{CI}=$ confidence interval; $\mathrm{IPC}=$ intermittent pneumatic compression; ISS $=$ Injury Severity Score; IV = intravenous; OR $=$ odds ratio; $\mathrm{RR}=$ risk ratio; $\mathrm{SC}=$ subcutaneously; $\mathrm{TID}=3$ times daily.

*Twice a day dosing.

$\dagger$ Dosing every 8 hours.

$\ddagger$ Intermittent pneumatic compression. 
Table 2: Cochrane Collaboration Tool for assessing risk of bias for randomized controlled trials

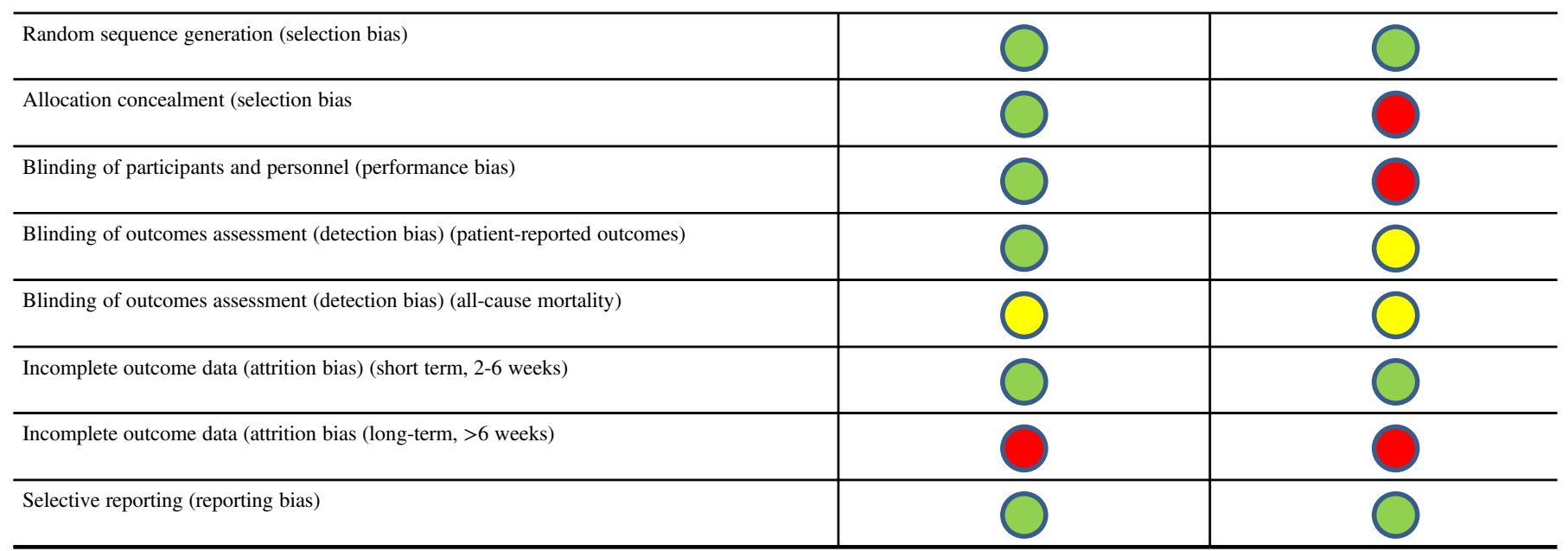

Low risk of bias

High risk of bias

Unsure risk of bias

mortality. Decisions regarding VTEp timing are largely based on individual experience because ACCP, BTF, and EAST guidelines are unable to support any recommendations regarding timing of VTEp initiation other than recommending that VTEp be initiated once hemorrhagic risk decreased. ${ }^{22,24}$

The efficacy of chemoprophylaxis in preventing VTE in hospitalized patients is well established. ${ }^{44}$ In trauma patients, delaying VTEp for 72 hours after injury doubles the risk of VTE, ${ }^{17}$ whereas delaying VTEp 96 hours after injury increases the risk threefold. ${ }^{45}$ In our review, four studies demonstrated that early administration of VTEp is associated with decreased rates of VTE ${ }^{17,41,46,47}$ whereas another seven studies identified VTE rates of only $0.0 \%$ and $2.0 \%$ in patients given VTEp within 24 to 72 hours of injury. This rate is much lower than commonly documented rates of VTE in TBI patients, further that early administration of VTEp reduces incidence of VTE. Three studies, however, found rates of VTE ranged from $7.3 \%$ to $14 \%$, despite administering VTEp within 72 hours of injury. ${ }^{21,54,57}$ Although rates of VTE in these three studies are relatively high despite early VTEp administration, patients in these studies were subject to routine screening with Doppler ultrasound; therefore, clinicians likely discovered subclinical DVTs that might not have been identified in other studies.

There are no consensus guidelines to recommend preferred agent, dose, or timing of chemical VTEp. The BTF guidelines suggest either UFH or LMWH because both are efficacious ${ }^{24}$; therefore, the decision of whether to use UFH or LMWH is largely based on practitioner and institution preference. LMWH has demonstrated superiority in PE prevention compared with UFH in large studies, however. For instance, a large, multicenter retrospective study on trauma patients demonstrated that LMWH was associated with nearly one-half the rate of PEs compared with UFH. ${ }^{58}$ Additionally, LMWH is associated with decreased rates of heparin-induced thrombocytopenia. ${ }^{59}$ As such, many trauma centers, including ours, favor LMWH as the agent of choice for chemical prophylaxis; however, UFH has a shorter half-life and is more easily reversed and thus may be the preferred agent when risk of hemorrhagic TBI progression is at stake. Clearly, there is a need for large-scale randomized trials to definitively investigate the potential benefits of LMWH compared with UFH in the setting of TBI.

Our primary objective was to determine if administering VTEp within 24 to 72 hours of TBI is safe or is dangerously associated with intracerebral hemorrhagic progression. Although the majority of evidence is retrospective, our study demonstrated that the practice of early VTEp administration after TBI is generally safe. Specifically, our review identified 18 studies that demonstrated that administering VTEp to patients with stable injury demonstrated by repeat head CT scan does not lead to progression of TBI. For example, Farooqui et $\mathrm{al}^{46}$ initiated a protocol in which all patients with TBI received either Enoxaparin or UFH 24 hours postinjury as long as patients were without coagulopathy or progressive hemorrhage seen on repeat head CT scan. Forty-eight to 72 hours after initiation of VTEp, all patients received a third CT scan of the head to assess for TBI progression. Investigators found that there were no significant differences in Glasgow Coma Scale (GCS) or hemorrhagic progression between patients who received early and late VTEp. The investigators also found that patients who received early VTEp had significantly shorter hospital stays (12.3 days vs 7.4 days, $\mathrm{p}<0.05$ ), shorter intensive care unit stays (9.2 days vs 5.0 days, $\mathrm{p}<0.001)$, and fewer VTEs $(5.6 \%$ vs $0.0 \%, \mathrm{p}<0.01)$ than those who were not included in the early VTEp protocol. However, the early (protocol) group had lower overall Injury Severity Scores than the nonprotocol group, thus potentially confounding these results. Similarly, Saadeh et al ${ }^{10}$ conducted a retrospective review of 122 TBI patients who were administered VTEp with LMWH 24 to 48 hours after stable head CT. Investigators found that none of the patients who received 
Table 3: Newcastle-Ottawa Scale Assessment of bias for cohort studies

\begin{tabular}{|c|c|c|c|c|c|c|c|c|c|}
\hline Study & $\begin{array}{l}\text { Representativeness } \\
\text { of cohort }\end{array}$ & $\begin{array}{c}\text { Selection of } \\
\text { nonexposed } \\
\text { cohort }\end{array}$ & $\begin{array}{l}\text { Ascertainment } \\
\text { of exposure }\end{array}$ & $\begin{array}{l}\text { Demonstration that outcome wasn't } \\
\text { present at start of study }\end{array}$ & Comparability & $\begin{array}{l}\text { Assessment } \\
\text { of outcome }\end{array}$ & $\begin{array}{l}\text { Was follow-up } \\
\text { long enough? }\end{array}$ & $\begin{array}{l}\text { Was follow-up } \\
\text { adequate? }\end{array}$ & $\begin{array}{l}\text { NOS quality score } \\
\text { (Sum of starts) }\end{array}$ \\
\hline $\begin{array}{c}\text { Kim et al. } \\
2002\end{array}$ & • & $\cdot$ & • & - & $\bullet$ & • & & & 7 \\
\hline $\begin{array}{l}\text { Norwood } \\
\text { et al. } 2002\end{array}$ & • & & - & - & & • & & & 4 \\
\hline $\begin{array}{l}\text { Kliendienst } \\
\text { et al. } 2003\end{array}$ & • & & • & • & & $\bullet$ & & & 4 \\
\hline $\begin{array}{l}\text { Lin et al. } \\
2013\end{array}$ & - & - & • & • & & $\bullet$ & & & 5 \\
\hline $\begin{array}{l}\text { Levy et al. } \\
2010\end{array}$ & • & • & • & • & •• & • & & & 7 \\
\hline $\begin{array}{l}\text { Depew et al. } \\
2008\end{array}$ & - & & - & - & & • & & & 4 \\
\hline $\begin{array}{l}\text { Dudley et al. } \\
2010\end{array}$ & • & • & • & • & & $\bullet$ & & & 7 \\
\hline $\begin{array}{l}\text { Norwood } \\
\text { et al. } 2008\end{array}$ & • & - & - & - & & - & & & 5 \\
\hline $\begin{array}{l}\text { Koehler et al. } \\
2011\end{array}$ & - & • & • & • & & $\bullet$ & & & 5 \\
\hline $\begin{array}{l}\text { Callcut et al. } \\
2011\end{array}$ & - & - & - & - & •• & • & • & • & 9 \\
\hline $\begin{array}{l}\text { Salottolo } \\
\text { et al. } 2011 \\
\end{array}$ & • & • & • & • & •• & • & & & 7 \\
\hline $\begin{array}{l}\text { Kwaitt et al. } \\
2012\end{array}$ & - & - & - & • & •• & • & & & 7 \\
\hline $\begin{array}{l}\text { Scudday } \\
\text { et al. } 2011\end{array}$ & • & • & • & • & •• & • & & & 7 \\
\hline $\begin{array}{l}\text { Nickele et al. } \\
2013\end{array}$ & • & - & - & - & & - & & & 5 \\
\hline $\begin{array}{l}\text { Minshall } \\
\text { et al. } 2011\end{array}$ & • & • & - & - & & • & & & 5 \\
\hline $\begin{array}{l}\text { Saadeh et al. } \\
2012\end{array}$ & • & - & - & - & & $\cdot$ & & & 5 \\
\hline $\begin{array}{l}\text { Farooqui } \\
\text { et al. } 2013 \\
\end{array}$ & • & $\cdot$ & - & - & & $\cdot$ & & & 5 \\
\hline $\begin{array}{l}\text { Kim et al. } \\
2014\end{array}$ & - & - & - & - & & $\bullet$ & & • & 6 \\
\hline
\end{tabular}

Full mark for Newcastle-Ottawa Scale was 9 points. Scores $\geq 7$ were considered high quality.

* The corresponding paper scores one point in this category.

记 $\quad * *$ The corresponding paper scores two points in this category. 


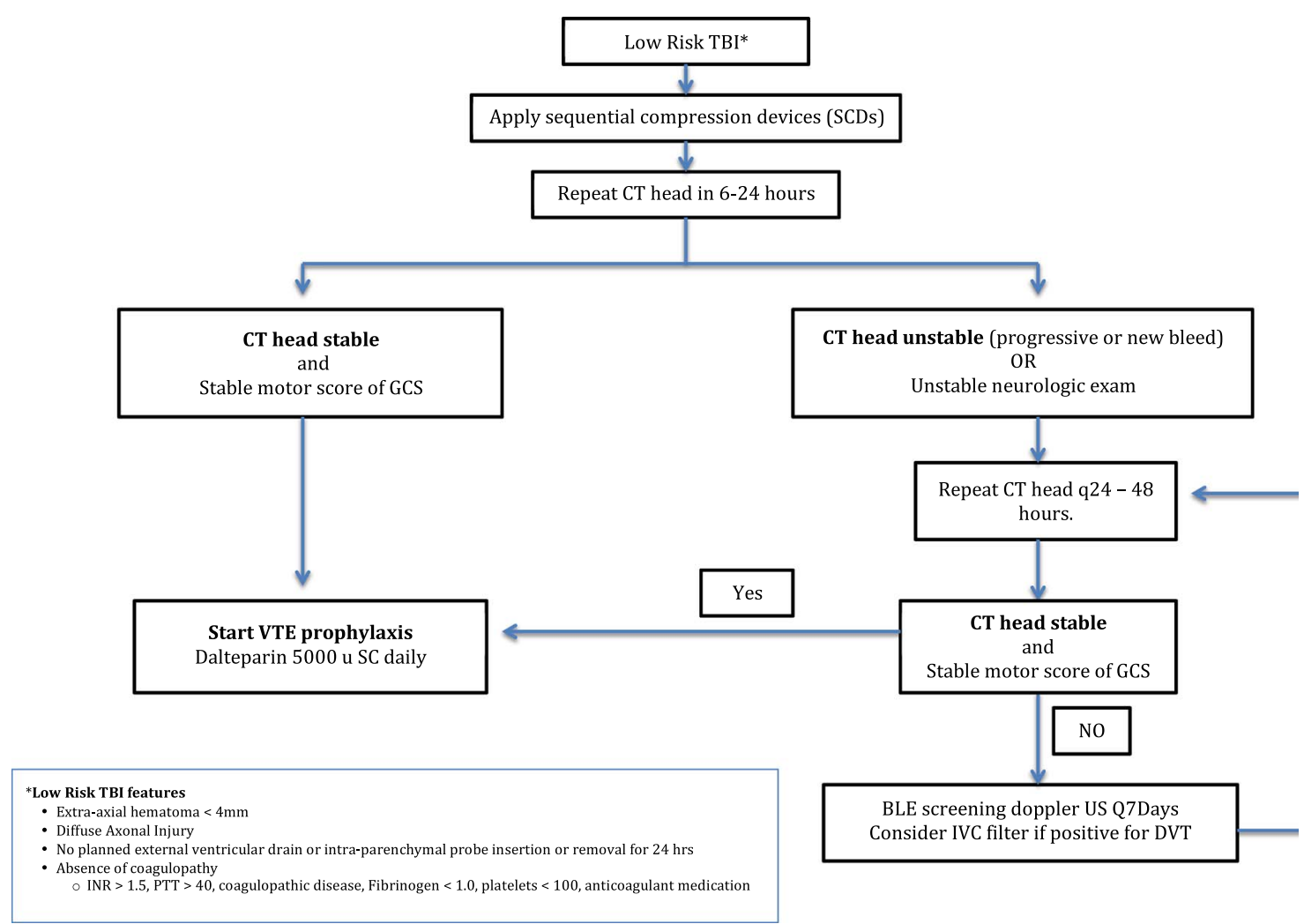

Figure 2: Clinical Practice Guidelines for Venous Thromboembolism Prophylaxis in Patients with low risk TBIs.

VTEp after TBI developed hemorrhagic progression; however, results from this study must be interpreted with caution because only $49.5 \%$ of eligible patients actually received VTEp within the 24- to 48-hour window. In a larger retrospective analysis study, Scudday et $\mathrm{al}^{47}$ found that 242 of 402 TBI patients had VTEp administered 24 to 72 hours after stable head CT scans. In fact, patients administered early VTEp had significantly lower GCS and Abbreviated Injury Scale scores than those not administered VTEp. Interestingly, despite having more severe brain injury, there was no significant increase in hemorrhagic progression between patients receiving early VTEp (3\% rate of progression) and patients not receiving VTEp (6\% rate of progression).

The potential devastating morbidity and mortality associated with hemorrhagic TBI progression makes performing randomized prospective trials on VTEp initiation in TBI patients practically and ethically challenging; however, we did identify two prospective studies. A randomized, double-blinded, noninferiority clinical trial conducted by Phelan et $\mathrm{al}^{49}$ demonstrated that administering VTEp within 48 hours of injury was associated with a TBI progression rate of $5.9 \%$, which was not significantly different from placebo. The authors loosely defined TBI progression as a finding on repeat CT scans of the head of "worse" hemorrhage compared with the initial CT scan of the head. Interestingly, all radiographic TBI progressions were subclinical. The authors of this study limited their subjects to patients with TBIs deemed "low risk" for rebleeding, which were subdural hematomas or epidural hematomas smaller than $8 \mathrm{~mm}$, intraventricular hemorrhage smaller than $2 \mathrm{~cm}$, small contusions, and small subarachnoid hemorrhage with normal CT angiogram of the head and neck vessels. This criteria, known as the Berne-Norwood Criteria, was conceived by Norwood et al in 2008. ${ }^{6}$ In their prospective cohort study, the authors enrolled "low-risk" TBI patients to receive VTEp within 48 hours of admission. Hemorrhagic progression was found in $18(3.4 \%)$ patients after starting VTEp. Ten of the 18 total hemorrhagic transformations and five of the six clinically significant hemorrhagic transformations were from protocol violations. Had the protocol violations been withheld from the analysis, the rate of TBI progression after VTEp would have dropped to $1.8 \%$. This study thus demonstrated that, in low rebleeding risk patients (as defined by the Berne-Norwood Criteria), VTEp can be administered safely within 48 hours of admission.

It is difficult to ascertain the natural history of TBIs and the baseline rate of hemorrhagic progression that occurs regardless of VTEp administration. For example, a retrospective cohort study by Kwiatt et $\mathrm{al}^{41}$ divided TBI patients into those who had received VTEp within 48 hours of admission, 48 hours to 7 days postadmission, and more than 7 days postadmission. The authors found that radiologic TBI progression occurred in $24 \%$ of patients who were not administered VTEp and that the rates of hemorrhagic progression did not increase once VTEp was given. In fact, there was no difference in the rate of hemorrhage progression after receiving VTEp regardless of when it was initiated. Of the patients who received VTEp within 48 hours, $22 \%$ had hemorrhage progression, which was similar to the rate of hemorrhagic progression in patients who had received LMWH 7 days postinjury, suggesting that timing of VTEp has no effect on TBI progression in this cohort. In total, $9.9 \%$ of patients with a stable repeat CT scan of the head before initiation of VTEp had progression after receiving 
VTEp. In this series, multivariate analysis implicated VTEp as the strongest risk factor for hemorrhagic progression (odds ratio [OR], 2.41). This study included patients who required emergency neurosurgical intervention, which may explain the high baseline progression rate of 24\%; however, deciphering which patients had TBI progression secondary to the natural history of TBIs regardless of LMWH is challenging.

That subtle hemorrhagic progression is frequently seen in repeat head CT scans indicates that subclinical progression is likely part of the natural history of TBIs. Furthermore, as CT scanning technology continues to improve, we are likely to continue identifying even more subtle changes in cerebral hemorrhage morphology. As such, the baseline rates of radiographic TBI progression in the absence of VTEp are variable. For example, Saddeh et $\mathrm{al}^{10}$ reported baseline hemorrhagic progression rate of $13.9 \%$, Norwood et al $(2002)^{55}$ found a baseline progression rate of $19 \%$, Callcut et $\mathrm{al}^{53}$ found baseline progression rate at $6.25 \%$, Scudday et $\mathrm{al}^{47}$ reported a baseline progression rate of $6 \%$, Phe$\operatorname{lan} 2012^{51}$ found a rate of $3.5 \%$, and Norwood et al $(2008)^{6}$ determined that $8.3 \%$ of their subjects had progression at baseline. One potential explanation for the variance in baseline hemorrhagic progression is that timing of a second CT scan of the head differs between studies. It is quite plausible that a repeat CT scan of the head performed earlier is more likely to demonstrate baseline hemorrhagic progression than one that is performed later after the traumatic event. However, although the rates of baseline TBI instability vary according to the literature, it may be acceptable to deem VTEp "safe" if its administration does not cause the rate of TBI progression to rise significantly over the baseline.

Ultimately, data from our review indicate that early VTEp is safe in patients with TBI; however, most of the high hemorrhagic risk patients have been excluded from analysis. As a result, there is insufficient evidence to generalize VTEp safety in all TBI patients. We have created a preliminary CPG for use in patients with TBI and low hemorrhagic risk features (Figure 2). This CPG is based on our systematic review of the literature and expert consultations with intensivists, neurosurgeons, and trauma surgeons at a Canadian Level-1 trauma center. The CPG, which has not yet been implemented, places patients into two protocol arms: high hemorrhagic risk and low hemorrhagic risk. The CPG highlights the need for clinicians to weigh hemorrhage and thrombosis risk on a patient-by-patient basis. Early and systematic identification of those patients with low risk of delayed bleeding would allow early VTEp initiation and therefore reduce the risk of VTE in a substantial segment of the TBI population. Conversely, early classification of patients into a high hemorrhagic risk category would allow us to minimize the risk of iatrogenic TBI progression resulting from anticoagulation. Studies have shown that elevated prothrombin time and international normalized ratio, antiplatelet medications, and prior anticoagulants are risk factors for progression. ${ }^{60-62}$ As a result, our CPG defines high hemorrhagic risk TBIs as extra axial hematomas $>4 \mathrm{~mm}$, diffuse axonal injury, TBIs requiring neurosurgical intervention, and TBIs occurring in patients with an international normalized ratio $>1.5$, a partial thromboplastin time $>40$ seconds, a platelet count of $<100 \times 10^{9}$ /liter, a known coagulopathy, and patients taking oral anticoagulants and/or anti-platelets medications. Patients with low hemorrhagic risk features and stable TBI would be allocated to a treatment pathway that supports administering VTEp with dalteparin 5000 units subcutaneously within 24 hours of injury.
Patients with unstable TBI and/or high hemorrhagic risk features would be shunted into a pathway that prioritizes mechanical VTEp, promotes early detection of DVT, and facilitates ongoing assessment hemorrhage risk based on serial head CTs. The BTF, EAST, and ACCP guidelines support the use of mechanical thromboprophylaxis with intermittent pneumatic compression whenever possible. $^{22,24}$ Therefore, all patients in the high-risk arm of the CPG would be administered mechanical thromboprophylaxis. Our CPG would also recommend screening for DVTs clinically on daily rounds and every 7 days with Doppler ultrasound in patients in the high-risk arm in the hopes of more quickly identifying subclinical DVTs. Last, the EAST guidelines make Level III recommendations to consider IVC filters in trauma patients who cannot receive anticoagulation ${ }^{22}$; therefore, our CPG also recommends consideration of an IVC filter if a DVT is found. Our next objective is to implement the CPG in TBI patients admitted to the intensive care unit and trauma services and compare rates of VTEs between historical controls and prospective patients treated in accordance with the CPG.

To our knowledge, this is the most extensive review of early VTEp administration in TBI patients, but there are several limitations of this study. For example, our meta-regression analysis did not detect a statistically significant relationship between timing of VTEp and hemorrhagic progression; however, because of study heterogeneity it is not possible to tell if the timing of VTEp truly has no influence on hemorrhagic progression or if the included studies are simply too heterogeneous to detect such an association. Additionally, most of the studies in our review did not specify which type of ICH (e.g. subarachnoid hemorrhage, epidural hematomas, subdural hematomas, contusions) were at higher risks of rebleeding following VTEp. We use TBI as an umbrella term for cerebral hemorrhage; however, in reality, TBI is a heterogenous group of injuries with different clinical features that are likely to respond differently to VTEp. Future research could focus on clarifying what characteristics of TBI are higher risk for progression following VTEp administration. We are also critical of using radiologic criteria to determine TBI progression; for example, dichotomizing TBI radiographically as either having "progressed" or "not progressed" fails to quantify the degree of expansion and association with clinical sequelae. Future research could focus on correlating radiographic TBI progression with clinical neurologic status. Also, lack of DVT screening in many of the studies has likely caused us to underestimate the true rates of VTE. Research on the utility of weekly DVT screening in trauma patients who are not administered VTEp could be an important area of future study because the longterm sequelae of subclinical DVTs may include postthrombotic syndrome, venous stasis, and delayed rehabilitation. ${ }^{62}$ Last, our preliminary CPG was developed based on a combination of findings identified from this review and expert opinion from a single center. Certainly, expert opinion from a single center represents the lowest grade of evidence, and the CPG itself is meant for low hemorrhagic risk patients, which limits its generalizability. Creating protocols for VTE prevention in high hemorrhagic risk TBI patients, such as those requiring neurosurgical intervention, was beyond the scope of this review, but is an important area for potential future research.

In conclusion, we have systematically reviewed the literature to determine that it is likely safe to administer VTEp 24 hours postinjury in clinically stable patients who have sustained a low hemorrhage risk TBI that has not progressed on repeat CT scan of the head. Future research is needed to identify specific risk factors for hemorrhagic progression that may preclude early VTEp 
administration. Until such factors are clearly delineated, we recommend a relatively conservative, yet consistent approach that includes administering VTEp within 24 hours of injury in patients with extra-axial hematomas smaller than $4 \mathrm{~mm}$ who are without coagulopathy or anticoagulant medications and have a radiographically and clinically stable TBI.

\section{ACKNOWLEDGMENTS}

We would like to thank Niki Baumann, Librarian at the College of Physicians and Surgeons of British Columbia Library, for her dedication and assistance with this review.

\section{Disclosures}

The authors have nothing to disclose.

\section{STATEMENT OF AUTHORSHIP}

JM: Literature search, study design, data collection, data interpretation, writing and critical revision. CD: Literature search, study design, data collection, data interpretation, critical revision. KD: Study design and critical revision. WC: Statistical analysis and critical revision. DCE: Study design and critical revision. MSS: Study design and critical revision. NG: Study design and critical revision. DEGG: Study design and critical revision. PG: Study design and critical revision. SMH: Principal investigator, study design, critical revision.

\section{REFERENCES}

1. Kim L, Holena D, Schuster J, Sims C, Levine J, Pascual JL. Early initiation of prophylactic heparin in severe traumatic brain injury is associated with accelerated improvement on brain imaging. J Emerg Trauma Shock. 2014;7:141-7.

2. Schaible E-V, Thal SC. Anticoagulation in patients with traumatic brain injury. Curr Opin Anaesthesiol. 2013;26:529-34.

3. Saiki RL. Current and evolving management of traumatic brain injury. Crit Care Nurs Cli N Am. 2009;21:549-59.

4. Spyropoulos AC, Hussein M, Lin J, Battleman D. Rates of venous thromboembolism occurrence in medical patients among the insured population. Thromb Haemost. 2009;102:951-7.

5. Ekeh AP, Dominguez KM, Markert RJ, McCarthy MC. Incidence and risk factors for deep venous thrombosis after moderate and severe brain injury. J Trauma. 2010;68:912-5.

6. Norwood SH, Berne JD, Rowe SA, Villarreal DH, Ledlie JT. Early venous thromboembolism prophylaxis with enoxaparin in patients with blunt traumatic brain injury. J Trauma. 2008;65:1021-7.

7. Geerts WH, Code KI, Jay RM, Chen E, Szalai JP. A prospective study of venous thromboembolism after major trauma. New Engl J Med. 1994;331:1601-6.

8. Phelan HA. Pharmacologic venous thromboembolism prophylaxis after traumatic brain injury: a critical literature review. J Neurotrauma. 2012;29:1821-8.

9. Gross AK, Norman J, Cook AM. Contemporary pharmacologic issues in the management of traumatic brain injury. J Pharm Pract. 2010;23:425-40.

10. Saadeh Y, Gohil K, Bill C, et al. Chemical venous thromboembolic prophylaxis is safe and effective for patients with traumatic brain injury when started 24 hours after the absence of hemorrhage progression on head CT. J Trauma Acute Care Surg. 2012;73:426-30.

11. Reiff DA, Haricharan RN, Bullington NM, Griffin RL, McGwin G Jr, Rue LW III. Traumatic brain injury is associated with the development of deep vein thrombosis independent of pharmacological prophylaxis. J Trauma. 2009;66:1436-40.
12. Foreman PM, Schmalz PGR, Griessenauer CJ. Chemoprophylaxis for venous thromboembolism in traumatic brain injury: a review and evidence-based protocol. Clin Neurol Neurosurg. 2014;123:109-16.

13. Praeger AJ, Westbrook AJ, Nichol AD, et al. Deep vein thrombosis and pulmonary embolus in patients with traumatic brain injury: a prospective observational study. Crit Care Resusc. 2012;14: 10-3.

14. Cherry RA, Nichols PA, Snavely TM, David MT, Lynch FC. Prophylactic inferior vena cava filters: do they make a difference in trauma patients? J Trauma. 2008;65:544-8.

15. Velmahos GC, Kern J, Chan LS, Oder D, Murray JA, Shekelle P. Prevention of venous thromboembolism after injury: an evidence-based report-part II: analysis of risk factors and evaluation of the role of vena caval filters. J Trauma. 2000;49:140-4.

16. Lu D, Mahmood A, Goussev A, et al. Atorvastatin reduction of intravascular thrombosis, increase in cerebral microvascular patency and integrity, and enhancement of spatial learning in rats subjected to traumatic brain injury. J Neurosurg. 2004;101:813-21.

17. Jamjoom AAB, Jamjoom AB. Safety and efficacy of early pharmacological thromboprophylaxis in traumatic brain injury: systematic review and meta-analysis. J Neurotrauma. 2013;30:503-11.

18. Tang ME, Lobel DA. Severe traumatic brain injury: maximizing outcomes. Mt Sinai J Med. 2009;76:119-28.

19. Levy AS, Salottolo K, Bar-Or R, et al. D. Pharmacologic thromboprophylaxis is a risk factor for hemorrhage progression in a subset of patients with traumatic brain injury. J Trauma. 2010;68:886-94.

20. Haddad SH, Arabi YM. Critical care management of severe traumatic brain injury in adults. Scand J Trauma Resusc Emerg Medicine. 2012;20:12.

21. Depew AJ, Hu J, Nguyen A, Driessen N. Thromboembolic prophylaxis is blunt traumatic intracranial hemorrhage: a retrospective review. Am Surg. 2008;74:906-11.

22. Rogers FB, Cipolle MD, Velmahos G, Rzycki G, Luchette FA. Practice management guidelines for the prevention of venous thromboembolism in trauma patients: the EAST Practice Management Guidelines Work Group. J Trauma. 2002;53:142-64.

23. Geerts WH, Bergqvist D, Pineo GF, et al. Prevention of Venous thromboembolism: American College of Chest Physicians evidence-based clinical practice guidelines. 8th ed. Chest. 2008;133(6Suppl):381S-453s.

24. Carney N, Totten AM, O'Reilly C, et al. Guidelines for the management of severe traumatic brain injury, fourth edition. Neurosurgery. 2017;80:6-15.

25. Moher D, Shamseer L, Clarke M, et al. Preferred reporting items for systematic review and meta-analysis protocols (PRISMA-P) 2015 statement. System Rev. 2015;4:1-9.

26. Phillips B, Ball C, Sackett D, Badenoch D, Straus S. Phillips: Oxford centre for evidence-based medicine. 2009. Available at: http://www.cebm.net/oxford-centre-evidence-based-medicine-levelsevidence-march-2009. Accessed September 24, 2015.

27. Higgins JPT, Altman DG, Gotzsche PC, et al. The Cochrane Collaboration's tool for assessing risk of bias in randomized trials. $\mathrm{Br}$ Med J. 2011;343:1-9.

28. Wells GA, Shea B, O'Connell D, Robertson J, et al. The NewcastleOttawa Scale (NOS) for assessing the quality of nonrandomized studies in meta-analyses. Ottawa Hospital Research Institute. 2011. Available at: http://www.ohri.ca/programs/clinical_ epidemiology/oxford.asp.

29. Algattas H, Huang J. Traumatic brain injury pathophysiology and treatments: early, intermediate, and late phases post-injury. Intl J Molec Sci. 2014;15:309-41.

30. Cowley NJ, da Silva EJ. Prevention of secondary brain injury following head trauma. Trauma. 2008;10:35-42.

31. Carlile M, Nicewander D, Yablon SA, Brown A, Brunner R, Burke D, Chae E, Englander J, Flanagan S, Hamond F, et al. Prophylaxis for venous thromboembolism during rehabilitation for traumatic brain injury: a multicenter observational study. J Trauma. 2009 Dec1-8.

32. Elliott B, Patel P, Matharu MS, Amos R, Machin S, Liu C, Greenwood R. Prophylaxis of venous thromboembolism during early inpatient rehabilitation after acquired brain injury: How guidelines change management. Disabil Rehabil. 2006;28:333-7. 
33. Grenander A, Bredbacka S, Rydvall A, et al. Antithrombin treatment in patients with traumatic brain injury. J Neurosurg Anesthesiol. 2001;13:49-56

34. Vergouwen MDI, Roos Y, Kamphusien PW. Venous thromboembolism prophylaxis and treatment in patients with acute stroke and traumatic brain injury. Curr Opin Crit Care. 2008; $14: 149-55$

35. Schoonman GG, Bakker DP, Jellema K. Low risk of late intracranial complications in mild traumatic brain injury patients using oral anticoagulation after an initial normal brain computed tomography scan: education instead of hospitalization. Eur J Neurol. 2014;21:1021-5.

36. Petruska DA. Prophylactic anticoagulation in patients with traumatic brain injury. J Am Coll Surg. 2011;213:809.

37. Phelan $\mathrm{H}$. Venous thromboembolism after traumatic brain injury. Semin Thromb Hemostasis. 2013;39:541-8.

38. Cullen N, Bayley M, Bayona N, Hilditch M, Aubut J. Management of heterotopic ossification and venous thromboembolism following acquired brain injury. Brain Inj. 2007;21:215-30.

39. Carlile MC, Yablon SA, Mysiw WJ, Frol AB, Lo D, Diaz-Arrastia R. Deep venous thrombosis management following traumatic brain injury: a practice survey of the traumatic brain injury model systems. J Head Trauma Rehabil. 2006;21:483-90.

40. Cothren CC, Smith WR, Moore EE, Morgan SJ. Utility of once-daily dose of low-molecular-weight heparin to prevent venous thromboembolism in multisystem trauma patients. World J Surg. 2006;31:98-104.

41. Kwiatt ME, Patel MS, Ross SE, et al. Is low-molecular-weight heparin safe for venous thromboembolism prophylaxis in patients with traumatic brain injury? A Western Trauma Association multicenter study. J Trauma Acute Care Surg. 2012;73:625-8.

42. Minshall CT, Eriksson EA, Leon SM, Doben AR, McKinzie BP, Fakhry SM. Safety and efficacy of heparin or enoxaparin prophylaxis in blunt trauma patients with a Head Abbreviated Injury Severity Score $>2$. J Trauma. 2011;71:396-400.

43. Lin M, Davis JV, Wong DT. Evaluation of heparin prophylaxis protocol on deep venous thrombosis and pulmonary embolism in traumatic brain injury. Am Surg. 2013;79:1050-3

44. Agnelli G, Piovella F, Buoncristiani P, et al. Enoxaparin plus compression stockings compared with compression stockings alone in the prevention of venous thromboembolism after elective neurosurgery. New Engl J Med. 1998;339:80-5.

45. Nathens AB, McMurray MK, Cuschieri J, et al. The practice of venous thromboembolism prophylaxis in the major trauma patient. J Trauma. 2007;62:557-63.

46. Farooqui A, Hiser B, Barnes SL, Litofsky NS. Safety and efficacy of early thromboembolism chemoprophylaxis after intracranial hemorrhage from traumatic brain injury. J Neurosurg. 2013;119:1576-82.

47. Scudday T, Brasel K, Webb T, et al. Safety and efficacy of prophylactic anticoagulation in patients with traumatic brain injury. J Am Coll Surg. 2011;213:148-53.

48. Kleindienst A, Harvey HB, Mater E, et al. Early antithrombotic prophylaxis with low molecular weight heparin in neurosurgery. Acta Neurochir. 2003;145:1085-91.

49. Koehler DM, Shipman J, Davidson MA, Guillamondegui O. Is Early venous thromboembolism prophylaxis safe in trauma patients with intracranial hemorrhage. J Trauma. 2011;70:324-9.

50. Nickele CM, Kamps TK, Medow JE. Safety of a DVT chemoprophylaxis protocol following traumatic brain injury: a single center quality improvement initiative. Neurocrit Care. 2012; 18:184-92.

51. Phelan HA, Wolf SE, Norwood SH, et al. A randomized, doubleblinded, placebo-controlled pilot trial of anticoagulation in low-risk traumatic brain injury. J Trauma Acute Care Surg. 2012;73:1434-41.

52. Salottolo K, Offner P, Levy AS, Mains CW, Slone DS, Bar-Or D. Interrupted pharmocologic thromboprophylaxis increases venous thromboembolism in traumatic brain injury. $\mathrm{J}$ Trauma. 2011;70:19-26.

53. Callcut RA, Hanseman DJ, Solan PD, et al. Early treatment of blunt cerebrovascular injury with concomitant hemorrhagic neurologic injury is safe and effective. J Trauma. 2011;72:338-46.
54. Dudley RR, Aziz I, Bonnici A, et al. Early venous thromboembolic event prophylaxis in traumatic brain injury with low-molecularweight heparin: risks and benefits. J Neurotrauma. 2010; 27:2165-72.

55. Norwood SH, McAuley CE, Berne JD, et al. Prospective evaluation of the safety of enoxaparin prophylaxis for venous thromboembolism in patients with intracranial hemorrhagic injuries. Arch Surg. 2002;137:696-702.

56. Kurtoglu M, Yanar H, Bilsel Y, et al. Venous thromboembolism prophylaxis after head and spinal trauma: Intermittent pneumatic compression devices versus low molecular weight heparin. World J Surg. 2004;28:807-11.

57. Kim J, Gearhart MM, Zurick A, Zuccarello M, James L, Luchette FA. Preliminary report on the safety of heparin for deep venous thrombosis prophylaxis after severe head injury. J Trauma. 2002;53:38-43.

58. Byrne JP, Geerts W, Mason SA, et al. Effectiveness of low-molecularweight-heparin versus unfractionated heparin to prevent pulmonary embolism following major trauma: a propensity-matched analysis. The J Trauma Acute Care Surg. 2017;82:252-62.

59. Greinacher A, Alban S, Omer-Adam MA, Weitschies W, Warkentin TE. Heparin-induced thrombocytopenia: A stoichiometry-based model to explain the differing immunogenicities of unfractionated heparin, low-molecular-weight-heparin, and fondaparinux in different clinical settings. Thromb Res. 2008;122:211-20.

60. Allison RZ, Nakagawa K, Hayashi M, Donavan DJ, Koenig MA. Derivation of a predictive score for hemorrhagic progression of cerebral contusions in moderate and severe traumatic brain injury. Neurocrit Care. 2017;26:80-6.

61. Zhang D, Gong S, Jin H, et al. Coagulation parameters and risk of progressive hemorrhagic injury after traumatic brain injury: systematic review and meta-analysis. BioMed Res Int. 2015; 2015:261825

62. Kelly J, Rudd A, Lewis RR, Hunt BJ. Screening for subclinical deep-vein thrombosis. QJM.. 2001;94:511-9.

\section{APPENDIX}

\section{Literature Search Strategy}

Literature search was performed with the assistance of the College of Physicians and Surgeons of British Columbia (CPSBC) librarian, using the CPSBC library access to EMBASE and MEDLINE databases.

\section{Medline (Through the PubMed Interface)}

"Brain Injuries/therapy"[Mesh]

AND

"Venous Thrombosis/prevention and control"[Mesh] OR

"Venous Thromboembolism/prevention and control"[Mesh] OR

"Anticoagulants"[Mesh] OR "Fibrinolytic Agents"[Mesh] AND

"Thrombolytic Therapy"[Mesh]

AND

english[lang] AND “Humans”[Mesh]

OR

"Brain Injuries"[Mesh]

AND

prophylaxis[tiab]

AND

"Venous Thrombosis/prevention and control"[Mesh] OR

"Venous Thromboembolism/prevention and control"[Mesh] OR

"Anticoagulants"[Mesh] OR "Fibrinolytic Agents"[Mesh] AND

"Thrombolytic Therapy"[Mesh] 
AND

english[lang] AND “Humans”[Mesh]

Limits: $1999+$

\section{EMBASE}

exp *traumatic brain injury/dm, dt, rh, th [Disease Management, Drug Therapy, Rehabilitation, Therapy]

\section{AND}

exp venous thromboembolism/pc [Prevention] OR exp anticoagulant agent/ OR exp fibrinolytic agent/ OR exp fibrinolytic therapy/ OR (anticoagula* OR prophyla* OR chemoprophyla* OR thromboprophyla* OR antithromb*).ti,ab.

NOT

(book or book series or conference abstract or conference paper or conference proceedings or "conference review" or short survey or trade journal).pt.

NOT

exp animal/ not exp human/

$$
\Rightarrow \text { NOT HELPFUL AT ALL! }
$$

\section{Second try:}

exp *traumatic brain injury/

AND

exp venous thromboembolism/pc [Prevention] OR exp anticoagulant agent/ OR exp fibrinolytic agent/ OR exp fibrinolytic therapy/ OR (anticoagula* OR prophyla* OR chemoprophyla* OR thromboprophyla* OR antithromb*).ti,ab.

NOT

(seizure prophylaxis OR preinjur* OR pre-injur*).ti,ab.

NOT

(book or book series or conference abstract or conference paper or conference proceedings or "conference review" or short survey or trade journal).pt.

NOT

exp animal/ not exp human/

$\Rightarrow$ much better!

Limits: English, 1999+, remove duplicates

\section{Search Strategies}

Some syntax that were used to describe search strategies:

AND means the article must contain at least one of the terms in the line above, and at least one of the terms below, in order to appear in the search results.

OR means the article must contain at least one of the terms in order to be found. Used for synonyms e.g. Venous Thrombosis OR Venous Thromboembolism
NOT means the article must not contain any of the search terms in the line below. e.g. NOT trade journal.pt. removes any results from trade journals from the search results.

The PubMed interface to Medline uses these tags:

[Mesh] means Medical Subject Headings. These are labels placed on each article to show what the article is about. These are highly effective, as they are "exploded" - that is, a search for "Anticoagulants"[Mesh] will search for more specific types of anticoagulants, such as Antithrombin Agents.

[tiab] means a search for this word in the title and abstract of all available articles

The EMBASE database uses different syntax:

exp something/ means that this term was searched as an Emtree Subject Heading. These are labels like Medical Subject Headings, just a different set because it's a different database. The "exp" part stands for explode, and works the same way as in PubMed, for example, exp venous thromboembolism/includes the search deep vein thrombosis/automatically.

asterisk (*) between the exp and the term means that one is searching for articles where this Subject Heading is considered to be the main focus of the article - that is, most if not all of the article is about this concept. It is particularly useful in the EMBASE database to eliminate irrelevant results.

(something).ti,ab. searches for the search word in the title and abstract of all available articles

asterisk $(*)$ means any ending for the word. E.g. anticoagula* will search for anticoagulant, anticoagulants, anticoagulation, anticoagulate, and so forth.

(something).pt. searches for particular document types. I often use this when I receive a large number of results, in order to remove items which are irrelevant (such as trade journals) and items which are hard to obtain (such as books and conference publications).

exp animal/ not exp human/ is a way of removing animal studies without removing articles about humans. Unlike Medline, EMBASE is not as consistent about labeling their Human studies, so this way removes any obviously irrelevant articles while leaving the unlabelled ones intact.

pre-injur*.ti,ab. searches for both "pre injury" and "pre-injury" automatically.

Remove duplicates is useful in the Ovid version of EMBASE (which I used), to find any articles which have been entered into the database twice, and remove the extra copies. 